\section{Sistema de vigilância alimentar e nutricional no Estado de São Paulo, Brasil: experiência da implementação e avaliação do estado nutricional de crianças}

\author{
Food and nutrition surveillance system in \\ the State of São Paulo, Brazil: experience of \\ the implementation and assessment of \\ children's nutritional condition
}

Sonia Isoyama Venâncio1

Renata Bertazzi Levy²

Silvia Regina Dias Médici Saldiva3

Lenise Mondini4

Maria Lúcia Rosa Stefanini 5

1-5 Núcleo de Investigação em Nutrição, Instituto de Saúde. Secretaria de Estado da Saúde. Rua Santo Antônio 590, $3^{\circ}$ andar. São Paulo, SP, Brasil. CEP: 01.314-000.

E-mail: soniav@isaude.sp.gov.br

\begin{abstract}
Objectives: to describe the strategy of the "SISVAN-SP" applicative implementation in the state of São Paulo and analyze the information generated by the System concerning the magnitude of nutritional deviations in children under five years old.

Methods: primary health units in the municipalities were in charge for collecting and entering data using an information system named "SISVAN-SP" an applicative developed by the Health Institute of the Health Secretariat of the State of São Paulo (SES/SP). Monthly available data of the system are: Health Regional Management, municipality, primary health unit, and medical file number, date of birth, date of visit, gender and classification of the children's nutritional condition. Children with weight/age < percentile 3 were considered undernourished and children with age/weight $\geq 97$ of the reference curve from the National Center of Health Statistics were considered in risk of overweight.

Results: malnutrition prevalence varied from $3.1 \%$ to $4.2 \%$ and overweight risk from 4.0 to $4.8 \%$ in the different municipalities; information quality and System coverage, even when variable were consistent with epidemiological information.

Conclusions: the "SISVAN-SP" applicative developed by SES/SP and used to monitor the nutritional condition of children up to five years old seen at the Primary Health Units was considered useful to assess and discuss aspects of the Food and Nutrition Surveillance System (SISVAN), such as data coverage and quality. Results support decision making aimed at children's weight prevention and control.

Key words Food and nutritional surveillance system, Children, Underweight, overweight, Data quality
\end{abstract}

\section{Resumo}

Objetivos: descrever a estratégia de implantação do aplicativo "SISVAN-SP" no Estado de São Paulo e analisar as informações geradas pelo Sistema quanto à distribuição e magnitude dos desvios nutricionais em crianças menores de cinco anos de idade.

Métodos: as Unidades Básicas de Saúde dos municípios foram responsáveis pela coleta, digitação e processamento de dados, utilizando um sistema de informações denominado "SISVAN-SP", aplicativo desenvolvido no Instituto de Saúde da Secretaria de Estado da Saúde de São Paulo (SES/SP). Os dados disponíveis mensalmente no sistema são: Direção Regional de Saúde, município, unidade básica de saúde, número do prontuário, data de nascimento, data do atendimento, sexo e classificação do estado nutricional das crianças. Foram consideradas desnutridas as crianças com peso/idade < percentil $3 e$ em risco de sobrepeso com peso/idade $\geq$ percentil $97 \mathrm{da}$ curva de referência do National Center of Health Statisics.

Resultados: a prevalência de desnutrição variou de $3,1 \%$ a 4,2\% e a de risco de sobrepeso 4,0 a 4,8\% nos diferentes municípios; a qualidade das informações e a cobertura do Sistema, ainda que variáveis, mostraramse consistentes com informações epidemiológicas.

Conclusões: o aplicativo "SISVAN-SP", desenvolvido pela SES/SP e utilizado para o monitoramento nutricional de crianças até cinco anos de idade, atendidas nas Unidades Básicas de Saúde, mostrou-se útil para avaliar e discutir aspectos do Sistema de Vigilância Alimentar e Nutricional (SISVAN), tais como cobertura e qualidade dos dados. Os resultados apontam para a definição de ações que visem a prevenção e controle de peso dessas crianças.

Palavras-chave Sistema de vigilância alimentar e nutricional, Crianças, baixo peso, Sobrepeso, Qualidade de dados 


\section{Introdução}

O Sistema de Vigilância Alimentar e Nutricional (SISVAN) corresponde a um sistema de coleta, processamento e análise contínuo dos dados de uma população, possibilitando diagnóstico atualizado da situação nutricional, suas tendências temporais e, também, dos fatores de sua determinação. Contribui para que se conheçam a natureza e a magnitude dos problemas de nutrição, caracterizando grupos sociais de risco e dando subsídios para a formulação de políticas e estabelecimento de programas e intervenções. ${ }^{1-3}$

No Brasil, embora experiências localizadas já estivessem ocorrendo desde meados da década de 70, o processo de implantação do SISVAN em nível nacional ganhou força nos anos 90, quando foi instituído pelo Ministério da Saúde por meio da Portaria 1.156 de 31/08/1990 e ainda com a inclusão da Vigilância Nutricional na Lei Orgânica do Sistema Único de Saúde (Lei n ${ }^{\circ}$ 8.080, de 19/09/1990, artigos $3^{\circ}, 6^{\circ}$ e $12^{\circ}$ ) e nas Normas Operacionais Básicas do Sistema Único de Saúde (SUS). 4

Cabe destacar que, em 1998, com a criação do programa de Incentivo de Combate às carências Nutricionais (ICCN), a implantação do SISVAN nos municípios da Federação passou a ser um dos requisitos básicos para o repasse de recursos financeiros federais mediante transferência dos recursos do Piso Assistencial Básico (PAB) (Portaria n 709 de 11/06/1999 do Ministério da Saúde). 5

Em 2001, o ICCN foi substituído pelo Programa Bolsa Alimentação, ampliando o número de beneficiários, e propondo como uma das ações a vigilância nutricional de gestantes e crianças (Medida Provisória 2.206 de 10/08/2001). 6 Em 2004, foi criado o Programa Bolsa Família, com a finalidade de unificação dos procedimentos de gestão e execução das ações de transferência de renda do Governo Federal. Nesse mesmo ano o Ministério da Saúde desenvolveu um aplicativo denominado de VAN (Vigilância Nutricional) a ser implantado em todos os municípios para o acompanhamento das famílias beneficiárias.

Posteriormente foram publicados o Decreto 5.209 de 17/09/2004 e a Portaria 2.246 de 20/10/2004 que:

[...] dispõe sobre as atribuições e normas para

a oferta e monitoramento das ações de saúde rela-

tivas às condicionalidades das famílias benefi-

ciárias do Programa Bolsa Família. (Brasil.

Decreto; 2004, p. 3). 7

A portaria 2.246 de 20/10/2004 que institui e divulga a implementação das ações de Vigilância
Alimentar e Nutricional no âmbito das ações básicas de saúde do Sistema Único de Saúde.8

No Estado de São Paulo, apesar das questões nutricionais não terem merecido destaque na agenda da Saúde até o final dos anos 90, a demanda crescente dos municípios habilitados no ICCN por instrumentos necessários para viabilizar a implantação do SISVAN levou à criação, em 2000, de um grupo composto por representantes das Coordenadorias de Planejamento em Saúde, do Interior e da Região Metropolitana da Grande São Paulo, do Conselho de Secretários Municipais de Saúde de São Paulo (COSEMS) e do Instituto de Saúde (órgão de pesquisa da Secretaria de Estado da Saúde de São Paulo - SES/SP), com a finalidade de institucionalizar e desenvolver os instrumentos necessários para viabilizar a implantação do SISVAN no Estado, resultando na elaboração de um aplicativo que foi denominado SISVAN-SP. ${ }^{9}$ Esse aplicativo consistiu em um sistema destinado à coleta de informações, processamento e análise de dados nutricionais de crianças menores de cinco anos de idade.

O presente artigo tem por objetivos descrever a estratégia de implantação do aplicativo SISVAN-SP no Estado de São Paulo e analisar as informações geradas pelo sistema quanto à distribuição e magnitude dos desvios nutricionais em crianças menores de cinco anos de idade.

\section{Métodos}

A estratégia de implantação no Sistema Único de Saúde de São Paulo de um aplicativo denominado SISVAN-SP, desenvolvido pelo Instituto de Saúde da Secretaria de Estado da Saúde de São Paulo, foi descrita a partir de análise de relatórios produzidos neste período.

Os dados sobre a cobertura do sistema foram calculados para os anos de 2001 e 2003, segundo as Regiões de Saúde do Estado, considerando o número de municípios que alimentaram o sistema pelo menos uma vez no ano.

Esse aplicativo, além dos dados gerais de identificação da Direção Regional de Saúde (DIR), Município, Unidade Básica de Saúde (UBS) e número do prontuário, fornecia também a data de nascimento, data do atendimento, sexo e a classificação do estado nutricional das crianças atendidas pelo SUS de São Paulo.

Os dados sobre estado nutricional de crianças referem-se às informações geradas pelo aplicativo em 2003. 
Para efeito de descrição da quantidade de crianças que alimentava o sistema mês a mês, considerou-se a primeira consulta no mês, para evitar que a mesma apresentasse duas medidas no mês, perfazendo um total de 2.222.493 crianças elegíveis para a análise. Para o cálculo de prevalência do estado nutricional foi considerada somente a primeira consulta da criança no ano, totalizando 1.060 .400 crianças.

No processo de consistência dos dados foram excluídas das análises as crianças que não apresentavam informações sobre "sexo" e/ou cuja idade encontrava-se acima de 60 meses (52.707 crianças, $2,3 \%$ ).

Para a avaliação do estado nutricional foram adotados os indicadores propostos para o acompanhamento do crescimento e desenvolvimento do Ministério da Saúde, 10 sendo consideradas com déficit de peso para idade as crianças classificadas abaixo do percentil 3 e em risco de sobrepeso aquelas classificadas igual ou acima do percentil 97 de peso/idade da curva de referência do National Center of Health Statistics (NCHS). A denominação aqui adotada "risco de sobrepeso" justifica-se pelo fato de o indicador utilizado não contemplar a distribuição do peso corporal em relação ao comprimento ou altura.

O peso corporal foi utilizado como medida antropométrica principal a ser analisada para compor os indicadores do estado nutricional infantil uma vez que é considerada uma das principais medidas utilizadas nos serviços de saúde para o acompanhamento do crescimento e desenvolvimento de crianças menores de cinco anos de idade. Além disso, não é incomum encontrar serviços de saúde que não dispõem de equipamento necessário e pessoal capacitado para a aplicação adequada das técnicas de coleta da medida da altura/comprimento da criança.

As prevalências dos desvios nutricionais foram descritas e analisadas segundo as variáveis sexo, idade e DIR.

Os dados foram analisados e processados utilizando o programa estatístico Stata 7.0.

\section{Resultados}

\section{Implantação do SISVAN-SP no Estado}

Devido à ausência de um sistema nacional para o gerenciamento de informações nutricionais, o Grupo Técnico de Apoio ao SISVAN da SES/SP desenvolveu um aplicativo com estas características para implantação no Estado de São Paulo.
Diversas etapas e pressupostos foram definidos antes que o aplicativo "SISVAN-SP" fosse desenvolvido e instalado. A construção desse instrumento partiu de necessidades identificadas pelo grupo e incorporou sugestões, tanto de conteúdos como de definição do fluxo das informações, durante o processo de sensibilização dos gestores regionais e municipais sobre a importância de um monitoramento da situação nutricional da população atendida no Sistema de Saúde.

O SISVAN/SP foi sendo incorporado pelos municípios de forma gradativa, respeitando-se as especificidades do sistema local e regional de saúde. Foi priorizado o acompanhamento de crianças menores de cinco anos, por se constituírem na parcela da população mais susceptível aos agravos nutricionais e atendendo as diretrizes da Política Nacional de Alimentação e Nutrição do Ministério da Saúde. Ainda, conforme recomendação do Ministério da Saúde, foi utilizado como ponto de partida para a introdução no sistema, somente um indicador relação do peso para a idade - para se estabelecer o diagnóstico e acompanhamento da situação nutricional. Fazia parte da estratégia de implementação a incorporação de outros indicadores no sistema, porém isso somente ocorreria após a consolidação do fluxo de informações com a análise dos resultados, aperfeiçoamento do sistema e da melhoria da qualidade na obtenção dos dados e nas respostas aos serviços de saúde com ações direcionadas à população atendida.

Testado no ano 2000 em alguns municípios, o aplicativo foi implantado na rede estadual em 2001, tendo sido alimentado de forma regular até o ano de 2003, quando se iniciou gradativamente o processo de substituição pelo aplicativo elaborado pelo Ministério da Saúde para todo o país. O sistema era alimentado, via eletrônica, pelos municípios que aderiram voluntariamente à proposta, os quais encaminhavam mensalmente os dados consolidados das suas UBS ao Instituto de Saúde.

No processo de implementação do SISVAN-SP houve a necessidade de capacitar a rede para a realização da avaliação antropométrica, tendo sido realizados treinamentos com essa finalidade nas 24 DIRs, envolvendo cerca de 1500 profissionais de saúde, no período de setembro a novembro de 2002.11 Adotou-se um mesmo padrão de referência para avaliação do estado nutricional das crianças no Estado, pois foram localizados vários municípios que, por questões históricas, utilizavam a curva de Marcondes, 12 a qual foi progressivamente substituída pelo padrão internacional de referência do crescimento do NCHS recomendado pelo working 


\section{Figura 1}

Evolução da adesão dos municípios ao SISVAN-SP nas Regiões de Saúde do Estado de São Paulo no período de 2001 a 2003.

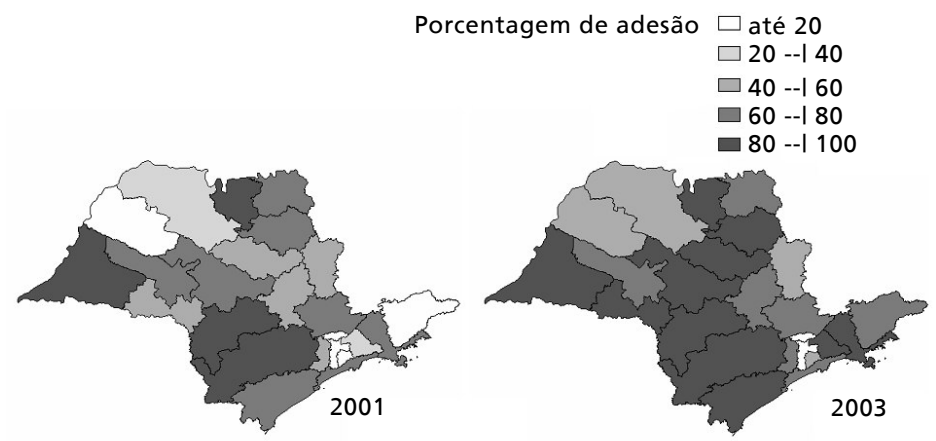

SISVAN-SP=Sistema de Vigilância Alimentar e Nutricional de São Paulo.

\section{Figura 2}

Prevalência (\%) de déficit ponderal e risco de sobrepeso em crianças menores de cinco anos de idade, mês a mês, atendidas nas UBS do SUS/SP no ano de 2003. SISVAN/SP.

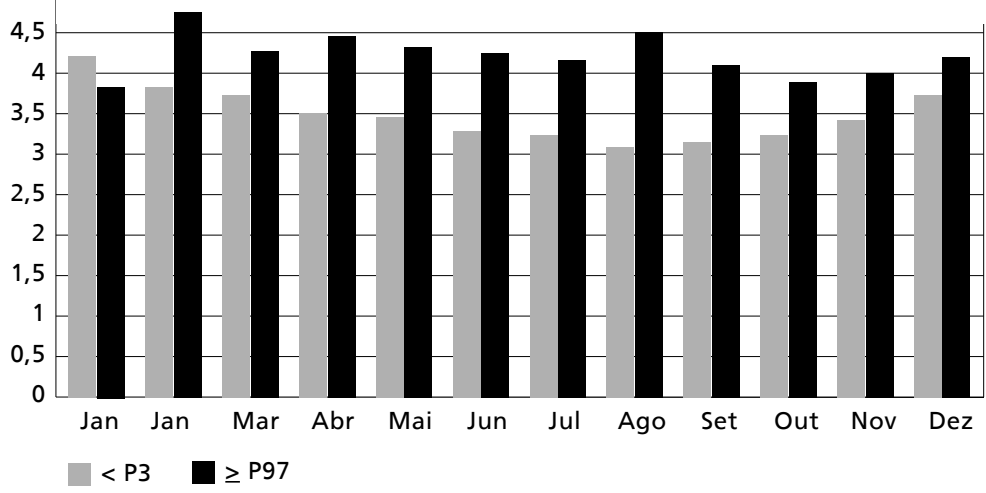

SISVAN/SP = Sistema de Vigilância Alimentar e Nutricional de São Paulo; UBS = Unidade Básica de Saúde; SUS/SP = Sistema Único de Saúde de São Paulo

Tabela 1

Número mensal de DIRs, de municípios, de UBS e de crianças menores de cinco anos de idade disponibilizados pelo aplicativo SISVAN-SP. São Paulo (Estado), 2003. *

\begin{tabular}{lcccc}
\hline Meses & DIR* (n) & Municípios* (n) & UBS (n) & Crianças (n) \\
\hline Janeiro & 22 & 422 & 1.588 & 190.896 \\
Fevereiro & 22 & 357 & 1.441 & 175.348 \\
Março & 19 & 375 & 1.511 & 192.345 \\
Abril & 21 & 400 & 1.554 & 208.735 \\
Maio & 20 & 380 & 1.506 & 203.157 \\
Junho & 18 & 281 & 1.228 & 154.238 \\
Julho & 20 & 364 & 1.503 & 191.909 \\
Agosto & 19 & 331 & 1.390 & 183.263 \\
Setembro & 20 & 382 & 1.457 & 204.259 \\
Outubro & 20 & 368 & 1.387 & 187.560 \\
Novembro & 21 & 371 & 1.385 & 175.972 \\
Dezembro & 20 & 384 & 1.387 & 154.811 \\
\hline
\end{tabular}

* Existem 24 DIRs e 645 municípios no Estado de São Paulo; UBS = Unidade Básica de Saúde; DIRs = Direção Regional de Saúde; SISVAN/SP = Sistema de Vigilância Alimentar e Nutricional de São Paulo. group da World Health Organization WHO.13 Os municípios receberam também apoio para a reprodução dos gráficos de crescimento, sendo que todas essas medidas propiciaram avanço em relação à qualidade das informações, especificamente quanto à padronização e comparabilidade dos dados gerados pelos diversos municípios do Estado.

A utilização do aplicativo no Estado foi crescente, sendo que, em 2001, 374 dos 645 municípios (58\%) encaminhavam dados para o Instituto de Saúde e, em 2003, o sistema estava implantado em $74,7 \%$ dos municípios (Figura 1).

A Tabela 1 mostra o número de DIR's, municípios e UBS que alimentaram o sistema e encaminharam seus bancos de dados ao Instituto de Saúde, no período de janeiro a dezembro de 2003. Verifica-se que das 24 Regionais existentes no Estado, houve adesão da maioria (22 no mês de janeiro e de 18 no mês de junho). Observa-se que há variação no número de municípios que enviaram informações mês a mês, sendo que a maior cobertura ocorreu no mês de janeiro $(65,4 \%$ dos municípios do Estado) e a menor cobertura no mês de junho (43,6\% dos municípios). Em face da forma irregular de alimentação do sistema pelos municípios, verifica-se também variação no número de UBS que registraram seus dados ao longo dos 12 meses. Chama a atenção o número expressivo de crianças que tiveram seus dados registrados mensalmente no período analisado, que variou de 154.238 a 208.735.

\section{Avaliação do estado nutricional das crianças}

Os dados analisados neste artigo correspondem ao conjunto das informações das crianças obtidas pelo sistema mensalmente no ano de 2003 , por considerar-se que nesse ano, após todos os treinamentos realizados sobre antropometria, o sistema gerou dados com melhor qualidade em relação aos anos anteriores.

A Figura 2 apresenta as prevalências de déficit de peso em relação à idade e de risco de sobrepeso entre as crianças menores de cinco anos, no período de janeiro a dezembro de 2003, atendidas pelo SISVAN/SP. A prevalência de déficit de peso/idade variou de $3,11 \%$ a $4,23 \%$ ao longo dos meses e a prevalência de risco de sobrepeso variou de 3,96\% a $4,75 \%$ no mesmo período. Observa-se que, ao longo dos doze meses, a frequiência de crianças com risco de sobrepeso supera àquela de crianças com baixo peso.

A Tabela 2 apresenta a distribuição das crianças segundo sexo e faixa etária, assim como a 
Prevalência (\%) de déficit de peso/idade e risco de sobrepeso, em crianças menores de cinco anos segundo sexo e faixa etária. SISVAN/SP. São Paulo (Estado), 2003.

\begin{tabular}{|c|c|c|c|c|}
\hline & & & $\%$ & $\%$ \\
\hline \multicolumn{5}{|l|}{ Sexo } \\
\hline Masculino & 538671 & 50,80 & 3,36 & 4,37 \\
\hline \multicolumn{5}{|c|}{ Faixa etária (meses) } \\
\hline $0-11$ & 381791 & 36,00 & $4,32 *$ & $3,85^{*}$ \\
\hline $12-23$ & 223510 & 21,08 & 4,63 & 3,41 \\
\hline $24-35$ & 177934 & 16,78 & 3,33 & 4,35 \\
\hline
\end{tabular}

* Teste de qui quadrado para tendência linear $(p<0,000)$; SISVAN/SP = Sistema de Vigilância Alimentar e Nutricional de São Paulo

prevalência dos desvios nutricionais de acordo com as referidas variáveis.

A distribuição de meninos e meninas atendidas pelo SISVAN foi homogênea, sendo que esta condição não foi observada em relação às faixas de idade. Neste caso, conforme o esperado, ocorreu concentração de crianças com menos de 24 meses de idade, sendo que pouco mais de $1 / 3$ delas com idade inferior a um ano.

As prevalências de déficit de peso/idade são bastante semelhantes entre as meninas e meninos, o mesmo ocorrendo em relação à magnitude do risco de sobrepeso, sendo que a condição de excesso supera a de déficit de peso, seja entre os meninos ou entre as meninas.

Em relação à idade, observa-se relação inversa entre prevalência de déficit de peso e faixa etária, enquanto a relação entre risco de sobrepeso e faixa etária é do tipo direta e positiva.

Vale ressaltar que somente entre as crianças menores de dois anos de idade a prevalência de déficit de peso/idade superou a de risco de sobrepeso.

A Tabela 3 apresenta a freqüência de baixo peso/idade e risco de sobrepeso segundo a região do Estado. Verifica-se que a situação nutricional das crianças menores de cinco anos diferiu entre as DIRs. A menor frequiência de déficit de peso/idade foi encontrada na região de Marília $(1,59 \%)$ e a maior freqüência na região de Piracicaba $(5,59 \%)$. Em relação ao risco de sobrepeso, a menor frequência foi verificada na região de Botucatu
Tabela 3

Prevalência (\%) de déficit de peso/idade e risco de sobrepeso, em crianças menores de cinco anos de idade segundo Diretorias Regionais de Saúde, (DIR.) SISVAN/SP. São Paulo (Estado), 2003.

\begin{tabular}{|c|c|c|c|}
\hline \multirow[t]{2}{*}{ DIR } & \multirow[t]{2}{*}{$\mathbf{N}$} & \multirow{2}{*}{$\begin{array}{c}\begin{array}{c}\text { Déficit de } \\
\text { peso/idade }\end{array} \\
\%\end{array}$} & \multirow{2}{*}{$\begin{array}{c}\begin{array}{c}\text { Risco de } \\
\text { sobrepeso }\end{array} \\
\%\end{array}$} \\
\hline & & & \\
\hline Santo André & 19.901 & 4,74 & 3,74 \\
\hline Mogi das Cruzes & 139.942 & 3,19 & 3,52 \\
\hline Osasco & 107.806 & 3,49 & 5,47 \\
\hline Araçatuba & 7.988 & 3,62 & 4,73 \\
\hline Araraquara & 36.184 & 3,94 & 5,06 \\
\hline Assis & 26.285 & 2,62 & 4,34 \\
\hline Barretos & 25.885 & 2,63 & 4,13 \\
\hline Bauru & 34.840 & 5,25 & 6,13 \\
\hline Botucatu & 26.589 & 3,25 & 2,78 \\
\hline Campinas & 81.126 & 3,19 & 4,60 \\
\hline Franca & 26.901 & 2,91 & 3,80 \\
\hline Marília & 38.714 & 1,59 & 3,85 \\
\hline Piracicaba & 41.082 & 5,59 & 4,70 \\
\hline Presidente Prudente & 52.325 & 2,35 & 5,10 \\
\hline Registro & 21.570 & 2,72 & 3,74 \\
\hline Ribeirão Preto & 30.602 & 4,21 & 4,60 \\
\hline Santos & 54.859 & 2,84 & 6,17 \\
\hline São João da Boa Vista & 26.005 & 2,30 & 3,35 \\
\hline São José dos Campos & 35.114 & 3,76 & 4,90 \\
\hline São José do Rio Preto & 33.175 & 3,19 & 3,14 \\
\hline Sorocaba & 168.954 & 5,10 & 3,37 \\
\hline Taubaté & 24.553 & 5,09 & 3,20 \\
\hline Total & 1.060 .400 & 3,65 & 4,28 \\
\hline
\end{tabular}

SISVAN/SP = Sistema de Vigilância Alimentar e Nutricional de São Paulo; DIR = Direção Regional de Saúde. 
$(2,78 \%)$ e a maior na baixada Santista $(6,17 \%)$. É interessante observar que em 16 das 22 DIRs analisadas a prevalência de risco de sobrepeso superou a prevalência de baixo peso, sendo que o inverso foi observado apenas nas regiões de Santo André, Botucatu, Piracicaba, São José do Rio Preto, Sorocaba e Taubaté.

\section{Discussão}

A experiência de implantação e desenvolvimento do SISVAN no Estado de São Paulo requer análise de duas ordens: uma relacionada à implantação propriamente dita e outra ao produto gerado a partir das informações sobre o estado nutricional infantil. Em uma dimensão técnica/operacional devem ser contemplados aspectos referentes à cobertura e qualidade dos dados, os quais, por sua vez, são fundamentais para conferir às informações confiabilidade e representatividade para a tomada de decisão, que é a manifestação concreta da vontade política. 14,15

No tocante à implantação do sistema, apesar de o Estado ter cumprido de forma tímida seu papel na indução de políticas de alimentação e nutrição na década de 90, a criação de um grupo técnico, com representação das diversas instâncias da SES/SP e do COSEMS significou um passo importante rumo à institucionalização da vigilância nutricional no Estado. Os municípios responderam, em sua maioria, de forma positiva à proposta de implantação do SISVAN-SP, o que veio ao encontro de uma necessidade criada a partir do ICCN.

Em relação à complexidade e às dificuldades para implantação do SISVAN, é possível, a partir da experiência paulista, levantar alguns pontos para reflexão e superação de obstáculos futuros.

Algumas questões devem ser analisadas, especialmente em relação à adesão dos municípios, envolvimento parcial/total das UBS e regularidade de alimentação do sistema. Os gestores municipais foram orientados a capacitar os profissionais das unidades básicas de saúde para preencher as planilhas com as informações de todas as crianças menores de cinco anos atendidas no serviço, e digitar mensalmente os dados. Os dados consolidados mostram que não houve adesão total dos municípios à proposta e que, nos municípios onde o sistema foi implantado, o encaminhamento dos bancos de dados foi realizado de forma descontínua. Verificaram-se ainda, em reuniões e treinamentos, vários questionamentos que apontaram para a necessidade de optar pelo envolvimento gradativo dos serviços, tais como: dificuldades dos profissionais em relação à pesagem das crianças em todas as consultas, ao registro sistemático das informações nas planilhas e à digitação dos dados. Nessas ocasiões, citava-se a grande quantidade de sistemas de informação implantados pelo Ministério da Saúde nos últimos anos, demandando grande esforço dos municípios para sua implementação. Esses problemas operacionais, somados à falta de "vontade política" de alguns gestores, poderiam explicar a não adesão de parte dos municípios e a dificuldade de implantação, em caráter universal, nas UBS.

É importante lembrar ainda que, em função das dificuldades para a implantação do sistema em caráter universal, os municípios podem priorizar, inclusive para responder a demandas do próprio Ministério, o acompanhamento de crianças beneficiárias de programas de suplementação alimentar, ou grupos populacionais residentes em áreas de grande vulnerabilidade social, o que pode enviesar a interpretação dos dados, superestimando a importância do baixo peso.

Todos esses aspectos, por terem implicações na cobertura e representatividade do sistema, precisam ser equacionados, a fim de tornar o SISVAN viável operacionalmente.

Embora seja inquestionável a necessidade de proceder à avaliação nutricional das crianças em todos os atendimentos, para seu acompanhamento individual, sugere-se, a partir da experiência relatada, outras formas de alimentação do sistema, como o registro no aplicativo da primeira consulta da criança no ano. Essa estratégia, adotada para o cálculo das prevalências de baixo peso e risco de sobrepeso, além de evitar, na análise, maior representação das crianças de menor idade, que em geral procuram com maior frequiência as UBS, reduziria substancialmente o trabalho dos municípios no tocante à digitação dos dados.

Cabe destacar que a implantação do SISVAN-SP representou importante contribuição para a qualificação da atenção à saúde da criança no Estado, uma vez que se identificou a ausência do acompanhamento do crescimento infantil em um número expressivo de unidades básicas de saúde e a necessidade de sensibilizar profissionais de saúde para resgatar essa prática. Além disso, os treinamentos realizados sobre antropometria em todas as regiões do Estado buscaram a qualificação dos dados gerados pelo sistema, a fim de garantir confiabilidade dos indicadores analisados.

Quanto à análise das informações sobre o estado nutricional infantil, verifica-se claramente que, embora o déficit de peso apareça de forma residual até os 23 meses, o risco de sobrepeso aparece com 
magnitude maior na população estudada, sendo que esse problema cresce à medida que aumenta a idade da criança.

As informações mais recentes sobre déficits antropométricos em crianças menores de cinco anos de idade provenientes de estudos populacionais na região Centro-Sul do país (dados não disponíveis para a região Sudeste) mostraram que $4,0 \%$ das crianças apresentavam déficit de peso/idade, grandeza semelhante à encontrada nesse estudo, apontando favoravelmente para a cobertura do sistema no Estado e a qualidade das informações. 16

Dados disponíveis para o município de São Paulo, também provenientes de inquéritos populacionais, revelam importância relativa crescente de excesso de peso em crianças menores de cinco anos. Em meados da década de 70 a proporção de baixo peso excedia em 1,7 vezes a de excesso de peso, enquanto que no último inquérito (1995/1996) ocorreu a inversão da magnitude dos desvios nutricionais e o excesso de peso passou a superar em seis vezes o baixo peso.17 Cabe pontuar aqui que a comparação deve ser feita com cautela, pois os indicadores que definem baixo peso e excesso de peso são oriundos da relação peso/altura, refletindo a distribuição do peso corporal em relação à estatura.

Um estudo de base populacional, realizado em cinco municípios do Estado de São Paulo em 2001, mostra também que a prevalência de obesidade de $6,6 \%$ foi superior ao déficit de altura, de $5 \% .18$

Os presentes resultados ilustram a transição nutricional em nosso país, onde não é mais possível que os formuladores de políticas restrinjam suas preocupações ao escopo dos distúrbios nutricionais relacionadas à escassez de alimentos. 17,19,20

\section{Referências}

1. Mason JB, Habitch J-P, Tabatabai H, Valverde V. Vigilância nutricional. Genebra: Organización Mundial de la Salud; 1984.

2. OPAS (Organización Panamericana de la Salud). Vigilancia alimentar y nutricional em las Americas. Washington, DC; 1989.

3. Ministério da Saúde. Secretaria de Atenção à Saúde. Departamento de Atenção Básica. Política nacional de alimentação e nutrição. 2. ed rev. Brasília, DF; 2003.

4. Stefanini MLR. Construindo o sistema de vigilância alimentar e nutricional do Estado de São Paulo. Bol Inst Saúde. 2000; 24: 16-18.

5. Engstrom EM, organizador. Sisvan: instrumento para o combate aos distúrbios nutricionais em serviços de saúde: diagnóstico nutricional. 2. ed. Rio de Janeiro: FIOCRUZ; 2002 .
Em suma, os resultados apresentados neste estudo podem contribuir para a reflexão sobre cumprimento do objetivo final do SISVAN em subsidiar decisões para o planejamento de políticas, gerenciamento e avaliação de programas de saúde e nutrição considerando a análise de aspectos técnico/operacionais que possam definir a agilidade do sistema para o fornecimento de respostas. Múltiplas razões podem frustrar o funcionamento de um SISVAN conforme apontado por diferentes autores, e podendo-se citar entre outras: a ausência de compromisso político dificultando a implementação do SISVAN, ainda que as políticas governamentais enfatizem a adoção de medidas na área nutricional; a complexidade na formulação e desenvolvimento dos Sistemas, tornando-os muitas vezes inoperáveis; a criação de estruturas técnico-administrativas exclusivas para o SISVAN, que passa a ser considerado como um núcleo isolado. 14,15

O SISVAN deveria provocar os gestores municipais e estaduais no sentido de orientar as políticas e as práticas de saúde, de acordo com a realidade epidemiológica, a qual mostra necessidade de planejamento de estratégias e ações intersetoriais que dêem conta da coexistência de distúrbios nutricionais de diferentes naturezas. Porém, é fundamental que aspectos técnicos/operacionais do Sistema que impliquem na agilidade das informações e na interpretação dos resultados sejam amplamente discutidos, a exemplo do relato da experiência paulista no que se refere à periodicidade do registro das informações no aplicativo e à consistência e análise dos dados, principalmente quando a proposta é universalizar o sistema no país, conforme orientações do Ministério da Saúde.

6. Brasil. Medida Provisória n. 2.206, de 10de agosto de 2001. Cria o Programa Nacional de Renda Mínima vinculado à Saúde: "Bolsa Alimentação". Diário Oficial da União. 2001: aug. 13; Sec E (154).

7. Brasil. Decreto n. 5.209 de 17 setembro de 2004. Regulamenta a Lei ${ }^{\circ} 10.836$ de 9 de janeirode 2004 que cria o Programa Bolsa Família e dá outras providências. Diário Oficial da União. 2004 set. 20; Sec. 1: 3

8. Brasil. Portaria n. 2246, de 18 de outubro de 2004: Institui e divulga orientações básicas para a implementação das Ações de Vigilância Alimentar e Nutricional, no âmbito das ações básicas de saúde do Sistema Único de Saúde - SUS em todo o território nacional. Diário Oficial da União. 2004 out. 18; Sec. 1: 28-9.

9. Cortez ECL, Figueiredo EM, Brandão NF. Desenvolvimento de um sistema de informações gerenciais para o Sistema de Vigilância Nutricional- SISVAN no estado de São Paulo. Bol Inst Saúde. 2002; 26: 9-11. 
10. Fagundes AA, Barros DC, Duar HA, Sardinha LMV, Pereira MM, Leão MM. SISVAN (Sistema de Vigilância Alimentar e Nutricional): orientações básicas para a coleta, o processamento, a análise de dados e a informação em serviços de saúde. Brasília, DF; 2004.

11. São Paulo [Estado]. Secretaria da Saúde. Instituto de Saúde. Curso de atualização em vigilância do crescimento: manual de orientação técnica. São Paulo; 2002.

12. Marcondes E. Curvas de crescimento. J Pediatr. 1986; 60: 241-9.

13. WHO (World Health Organization). Working Group. Use and interpretation of anthropometric indicators of nutritional status. Bull World Health Organ. 1986; 64: 929-41.

14. Arruda BKG. Sistema de Vigilância Alimentar e Nutricional: frustrações, desafios e perspectivas. Cad Saúde Pública. 1992; 8: 96-101.

15. Castro IRR. Vigilância alimentar e nutricional: limitações e interfaces com a rede de Saúde. Rio de Janeiro: FIOCRUZ; 1995.
16. Monteiro CA. Evolução da nutrição infantil nos anos 90 . In: Monteiro CA (organizador). Velhos e novos males da saúde no Brasil: a evolução do país e suas doenças. 2. ed aum. São Paulo: Hucitec; 2000. p. 375-92.

17. Monteiro CA, Conde WL. Tendência secular da desnutrição e da obesidade. Rev Saúde Pública. 2000; 34: 52-61.

18. Saldiva SRDM, Escuder MML, Venancio SI, Benício MHD'A. Prevalence of obesity in preschool children from five towns in São Paulo State, Brazil. Cad Saúde Pública. 2004; 20: 1627-32.

19. Monteiro CA, Benício MHD'A, Conde WL, Popkin BM. Shifting obesity trends in Brazil. European J Clin Nutr. 2000; 54: 1-5.

20. Batista Filho M, Rissin A. Transição nutricional no Brasil: tendências regionais e temporais. Cad Saúde Pública. 2003;19 (Supl 1): S181-91.

Recebido em 19 de julho de 2006

Versão final apresentada em 23 de abril de 2007

Aprovado em 30 de abril de 2007 